\title{
Improved pressure contour analysis for estimating cardiac stroke volume using pulse wave velocity measurement
}

\author{
Shun Kamoi ${ }^{*}$, Christopher Pretty ${ }^{1}$, Joel Balmer ${ }^{1}$, Shaun Davidson ${ }^{1}$, Antoine Pironet ${ }^{2}$, Thomas Desaive ${ }^{2}$, \\ Geoffrey M. Shaw ${ }^{3}$ and J. Geoffrey Chase ${ }^{1}$
}

\section{*Correspondence:}

shun.kamoi@pg.canterbury. ac.nz

${ }^{1}$ Department of Mechanical Engineering, University of Canterbury, Christchurch, New Zealand

Full list of author information is available at the end of the article

\begin{abstract}
Background: Pressure contour analysis is commonly used to estimate cardiac performance for patients suffering from cardiovascular dysfunction in the intensive care unit. However, the existing techniques for continuous estimation of stroke volume (SV) from pressure measurement can be unreliable during hemodynamic instability, which is inevitable for patients requiring significant treatment. For this reason, pressure contour methods must be improved to capture changes in vascular properties and thus provide accurate conversion from pressure to flow.
\end{abstract}

Methods: This paper presents a novel pressure contour method utilizing pulse wave velocity (PWV) measurement to capture vascular properties. A three-element Windkessel model combined with the reservoir-wave concept are used to decompose the pressure contour into components related to storage and flow. The model parameters are identified beat-to-beat from the water-hammer equation using measured PWV, wave component of the pressure, and an estimate of subject-specific aortic dimension. $\mathrm{SV}$ is then calculated by converting pressure to flow using identified model parameters. The accuracy of this novel method is investigated using data from porcine experiments $(\mathrm{N}=4$ Pietrain pigs, $20-24.5 \mathrm{~kg}$ ), where hemodynamic properties were significantly altered using dobutamine, fluid administration, and mechanical ventilation. In the experiment, left ventricular volume was measured using admittance catheter, and aortic pressure waveforms were measured at two locations, the aortic arch and abdominal aorta.

Results: Bland-Altman analysis comparing gold-standard SV measured by the admittance catheter and estimated SV from the novel method showed average limits of agreement of $\pm 26 \%$ across significant hemodynamic alterations. This result shows the method is capable of estimating clinically acceptable absolute SV values according to Critchely and Critchely.

Conclusion: The novel pressure contour method presented can accurately estimate and track SV even when hemodynamic properties are significantly altered. Integrating PWV measurements into pressure contour analysis improves identification of beat-tobeat changes in Windkessel model parameters, and thus, provides accurate estimate of blood flow from measured pressure contour. The method has great potential for overcoming weaknesses associated with current pressure contour methods for estimating SV. 
Keywords: Pressure contour analysis, Hemodynamic monitor, Stroke volume, Pulse wave velocity, Reservoir-wave pressure, Water hammer, Cardiovascular system, Physiological modelling, Windkessel model, Intensive care

\section{Background}

Stroke volume (SV) is an important physiological parameter for diagnosing and treating patients suffering from cardiovascular dysfunctions [1-4]. Accurately tracking changes in SV provides an in-depth picture of cardiovascular condition and response to therapy, thus enabling more optimal care $[5,6]$. Although SV measurements are increasingly seen as essential for correct clinical decisions, accurate continuous SV measurement requires highly invasive instrumentation, such as inserting an admittance catheter into the ventricle and is thus not clinically feasible.

At present, SV is continuously measured indirectly using continuous cardiac output monitors in the intensive care unit (ICU) [7, 8]. Non-invasive methods, such as esophageal Doppler and bio-impedance exist, but can be operator-dependent [9] and inaccurate due to the signal quality [10]. Moderately invasive methods, such as pressure contour analysis have been shown to be unreliable during hemodynamic instability [11]. The error comes from calibration methods involving use of fixed arterial properties, such as peripheral resistance, compliance, and vascular impedance [12], which certainly change as patient condition evolves and due to clinical interventions.

Inaccurate surrogate measures of SV could lead to misdiagnosis, incorrect clinical treatment/decisions, and/or misinterpretation of patient response to therapy. In the ICU, changes in the hemodynamic state are expected, and assuming constant hemodynamic parameters may not be suitable in many situations, such as fluid resuscitation [13] and/or inotrope therapy [14]. Therefore, there is a need for accurate and robust methods to estimate SV that are reliable even when hemodynamic properties are evolving rapidly.

This paper presents a novel pressure contour method for estimating SV. A three-element Windkessel model [15] combined with the reservoir-wave concept [16] are used to analyse the pressure contour. Windkessel parameters are identified using pressure and pulse wave velocity (PWV) measurements using a calibrated arterial diameter. Identified parameters are used to calculate flow from pressure measurements and SV is then calculated by integrating the estimated flow.

The distinct difference between the proposed method and the traditional methods [17, $18]$ is that PWV measurements are used to improve the capacity of pressure contour analysis. Furthermore, reservoir-wave separation was performed on the pressure waveform to accurately capture the changes in Windkessel parameters [19]. The separation provides more accurate information on which Windkessel parameter is responsible for the changes in the shape of pressure waveform.

Pressure contour analysis has traditionally been purely based on the morphology of the pressure waveform alone [20]. Consequently, the weaknesses associated with monitoring SV were always found during hemodynamic instability [21], which is the exact point where monitoring patients SV becomes essential. To overcome the limited information achievable from a single measurement, the presented method couples pressure and PWV measurements to improve the analysis of circulatory system. To investigate 
the accuracy of this new method, Bland-Altman plots are presented for data from porcine experiments, where beat-to-beat SV is estimated and compared against SV measured directly from an admittance catheter.

\section{Methods}

\section{Porcine experiments}

All experimental procedures, protocols and the use of data in this study were reviewed and approved by the Ethics Committee of the University of Liege Medical Faculty.

Experiments were performed on four healthy pigs weighing between 20 and $24.5 \mathrm{~kg}$ and a total of 13,409 heart beats were recorded. The pigs were premedicated with ketamine $(20 \mathrm{mg} / \mathrm{kg})$ and diazepam $(1 \mathrm{mg} / \mathrm{kg})$. Anesthesia was induced and maintained by a continuous infusion of sufentanil $(0.5 \mu \mathrm{g} / \mathrm{kg} / \mathrm{h})$ and sodium pentobarbital $(3 \mathrm{mg} / \mathrm{kg})$. The pigs were intubated via tracheotomy and ventilated using a GE Engstrom Carestation ventilator (GE healthcare, Chicago, United States).

Cardiovascular measurements were continuously recorded using Notocord-hem software (Notocord, Croissy-sur-Seine, France). Left ventricular pressures and volumes were measured using 7F micromanometer-tipped admittance catheters (Transonic Scisense Inc., Ontario, Canada) inserted into the ventricles through the right carotid artery. Central pressure waveform measurements were captured at the aortic arch and abdominal aorta with 7F pressure catheters (Transonic Scisense Inc., Ontario, Canada). Catheters for central pressure waveform measurements were inserted into the aortic arch through left carotid artery and the abdominal aorta through the femoral artery, respectively. All cardiovascular and respiratory data were recorded with the chest closed and sampled at $250 \mathrm{~Hz}$. The analysis in this study was performed using Matlab (version 2015a, The Mathworks, Natick, Massachusetts, USA).

\section{Experimental protocol}

The experiment comprised three different interventions intended to induce hemodynamic variations: (1) volume expansion by saline solution; (2) continuous infusion of the inotrope dobutamine; and (3) step-wise changes in positive end expiratory pressure (PEEP) recruitment manoeuvres (RM). For each pig the experiment started with administration of five rapid saline bolus of $180 \mathrm{ml}$ up to a total of $900 \mathrm{ml}$, except for Pig 4 where a total of $720 \mathrm{ml}$ of saline solution was given. After fluid administration, the pigs received a continuous infusion of dobutamine at a rate of either 2.5 or $5 \mu \mathrm{g} / \mathrm{kg} / \mathrm{min}$. In Pigs 3 and 4, further administration of a $180 \mathrm{ml}$ rapid bolus were given while continuing dobutamine infusion. The summary of interventions made for each pig are given in Table 1, and provide a wide range of induced hemodynamic changes.

Between each intervention, a RM was performed to induce changes in cardiac preload. An increased PEEP results in a smaller pressure gradient between the peripheral veins and the right atrium and decreases systemic venous return [22]. In addition, pulmonary circulation resistance is increased, and as a consequence, left ventricular SV is reduced [23]. RMs involved increasing PEEP with increments of $5 \mathrm{~cm} \mathrm{H}_{2} \mathrm{O}$ to a maximum of $20-25 \mathrm{~cm} \mathrm{H}_{2} \mathrm{O}$ and then reducing PEEP back to the original PEEP level of $5 \mathrm{~cm} \mathrm{H}_{2} \mathrm{O}$ in a step-wise manner. An example of the relationship between SV and PEEP are shown in Fig. 1. 
Table 1 Summary of interventions made for each pig in the experiment

\begin{tabular}{|c|c|c|c|c|c|c|c|c|}
\hline \multirow[t]{2}{*}{ Pig no. } & \multicolumn{5}{|c|}{$\begin{array}{l}\text { Volume } \\
\text { expansion }\end{array}$} & \multicolumn{2}{|c|}{$\begin{array}{l}\text { Dobutamine } \\
(\mu \mathrm{g} / \mathrm{kg} / \mathrm{min})\end{array}$} & \multirow{2}{*}{$\begin{array}{l}\text { Dobutamine }+ \\
\text { volume expansion } \\
180 \mathrm{ml}\end{array}$} \\
\hline & $180 \mathrm{ml}$ & $360 \mathrm{ml}$ & $540 \mathrm{ml}$ & $720 \mathrm{ml}$ & $900 \mathrm{ml}$ & 2.5 & 5 & \\
\hline 1 & $\checkmark$ & $\checkmark$ & $\checkmark$ & $\checkmark$ & $\checkmark$ & $\times$ & $\checkmark$ & $x$ \\
\hline 2 & $\checkmark$ & $\checkmark$ & $\checkmark$ & $\checkmark$ & $\checkmark$ & $\times$ & $\checkmark$ & $\times$ \\
\hline 3 & $\checkmark$ & $\checkmark$ & $\checkmark$ & $\checkmark$ & $x$ & $\checkmark$ & $x$ & $\checkmark$ \\
\hline 4 & $\checkmark$ & $\checkmark$ & $\checkmark$ & $\checkmark$ & $\checkmark$ & $x$ & $\checkmark$ & $\checkmark$ \\
\hline
\end{tabular}
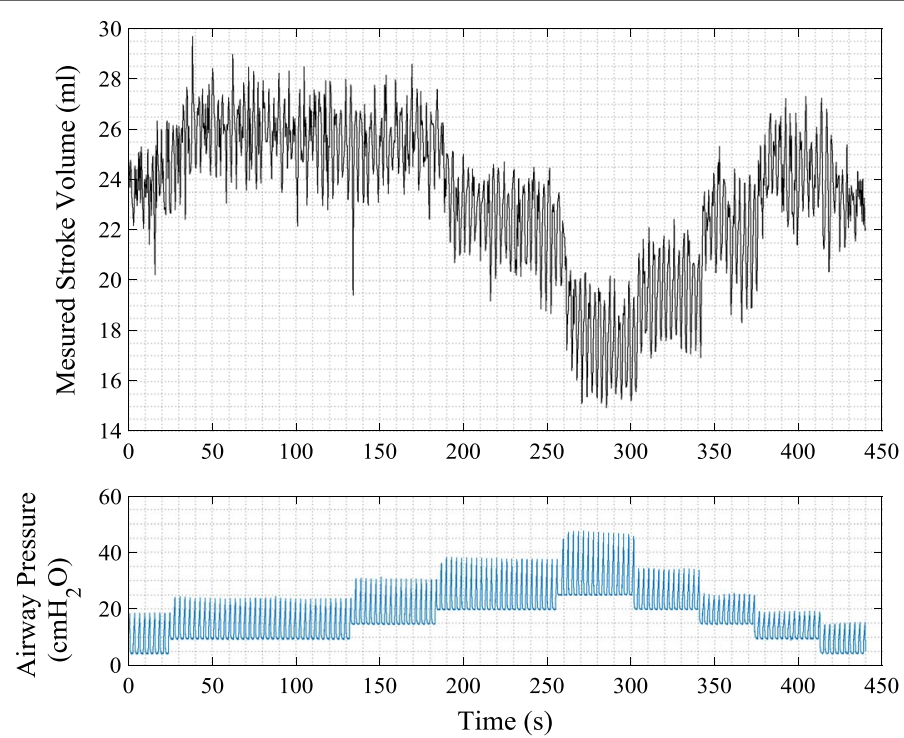

Fig. 1 Example of relationship between directly measured SV and PEEP from an experiment. Top panel measured SV from left ventricular admittance signal. Bottom panel measured airway pressure from mechanical ventilator

\section{Pulse wave velocity measurements}

The velocity of the pressure wave along the aorta was calculated using the aortic arc and abdominal pressure waveform measurements. The transit time of the pressure wave was measured by locating the 'foot' of the systolic rise in each measurement. In this study, the 'foot' of the pressure was identified as intersection of the tangent line along the maximum systolic pressure gradient and the horizontal line along the minimum pressure point. Example of the identified 'foot' in a single heart beat is shown in Fig. 2.

The distance between the two pressure catheters was measured on the body surface by approximating the catheter locations. This method may introduce error in the absolute value of PWV, but as the catheter locations were fixed throughout each experiment, PWV trends for a given pig were unaffected.

\section{Detection of end systolic point}

This analysis requires the maximum negative gradient of the pressure waveform per beat, characterised by end systolic point (ESP) [24]. However, this condition is not robust 


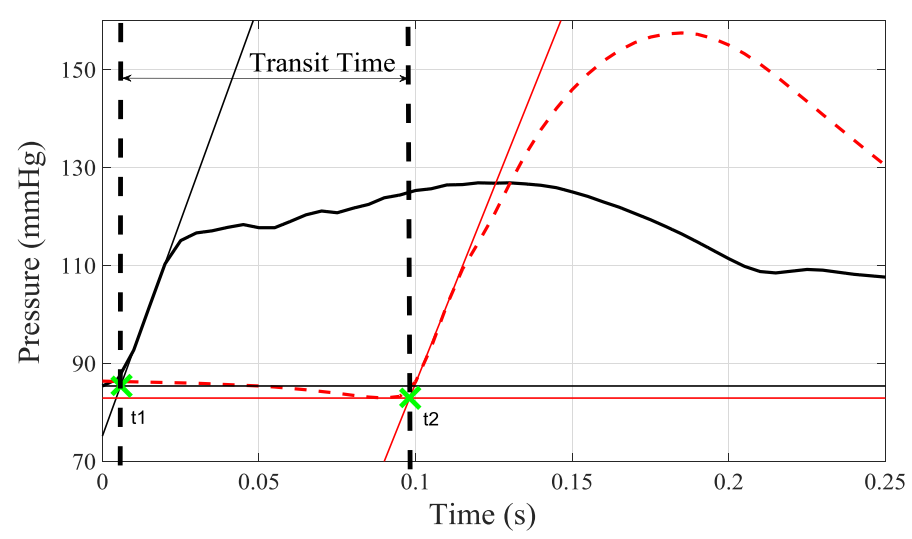

Fig. 2 Example of measured aortic arch (black) and abdominal aorta (dashed red) pressure waveforms. Thin lines represent tangent line along maximum pressure gradient and horizontal lines along minimum pressure point for each of the waveform. The green crosses represent the identified 'foot' of the pressure waveforms at times $t 1$ and $t 2$ for aortic arch and abdominal pressure, respectively. Transit time can be seen as the difference (t2-t1) between these time points

enough for typically measured pressure waveforms, which can have multiple inflection points and thus multiple minima in the $\mathrm{dP} / \mathrm{dt}$ waveform. An example is shown in Fig. 3.

Figure 3 shows multiple local and global minimum gradient points. In this specific case, the correct location of the ESP would be the second minimum gradient point and not the global minimum point. To avoid false detection of the ESP, which is required for calculating SV, a generic Weight Function (WF) was applied to the gradient of the pressure curve.

$$
\begin{aligned}
& W F(t)=\left(0.5-\left|0.5-\frac{H R . t}{60}\right|\right)^{2} \\
& E S P=\min \left(\frac{d P_{\text {measured }}}{d t} W F(t)\right)
\end{aligned}
$$

where HR is the heart rate.

This approach applies a greater weighting to points near the midpoint of the cardiac cycle and thus, enhances minima in the expected ESP location. This procedure is illustrated in Fig. 3.

\section{Pressure contour analysis}

A measured continuous abdominal pressure waveforms was used to estimate SV in this investigation. The continuous waveforms were first split into individual heart beats for the beat-to-beat pressure contour analysis. For each beat, a reservoir-wave separation was applied and the wave component of the pressure waveform was identified. Aortic characteristic impedance was then calculated by solving the water-hammer equation. Finally, the wave component of the pressure is used with the calculated value of aortic characteristic impedance to estimate beat-to-beat SV. 

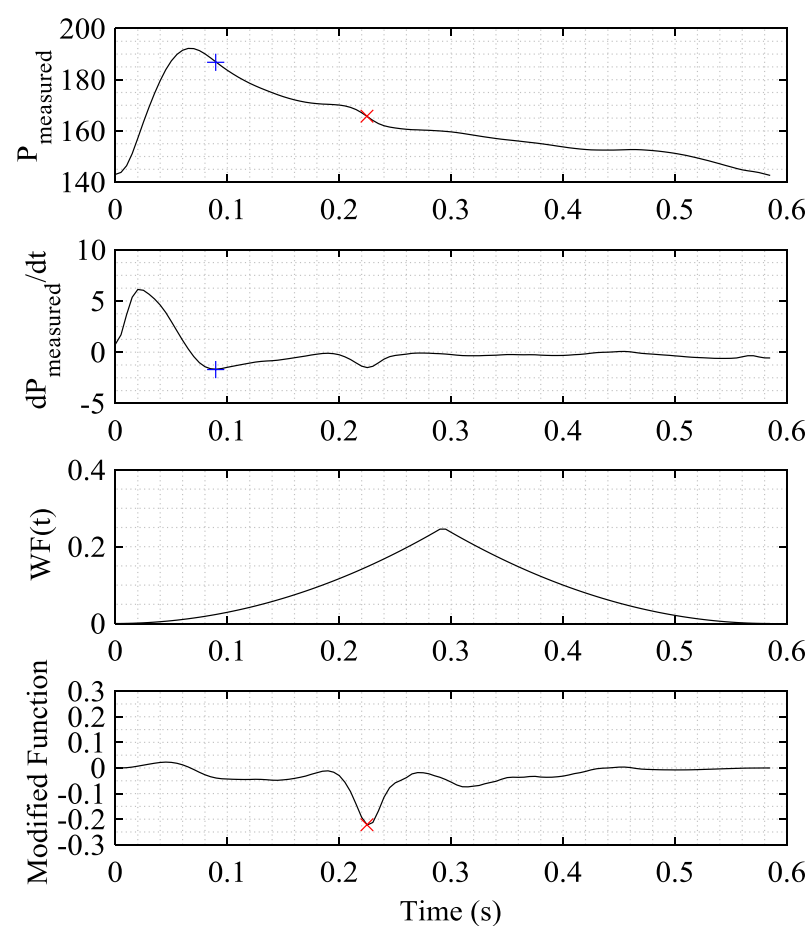

Fig. 3 Detected maximum negative gradient point $(b / u e+)$ and detected end systolic point using the modified function (red $x$ ) on a single beat pressure waveform. Top panel measured pressure waveform having multiple descending inflection points. Second panel pressure gradient $d P_{\text {measured }} / d t$ and detected global minimum, shown as blue cross. Third panel weight function WF(t) applied to $d P_{\text {measured }} / d t$. Bottom panel modified function showing product of $d P_{\text {measured }} / d t$ and WF(t), where the red cross shows the identified location of the end systolic point

\section{Reservoir-wave separation}

The time-varying reservoir pressure proposed by Wang et al. [25] is used to analyse the pressure contour. This model interprets the measured pressure as a sum of two pressure components, reservoir pressure $P_{r e s}$, and excess pressure $P_{e x}$.

$$
P_{\text {measured }}(t)=P_{\text {res }}(t)+P_{\text {ex }}(t)
$$

$P_{\text {res }}$ represents the energy stored and released by the aortic compliance, and $P_{e x}$ is the wave component of the pressure waveform and represents the excess amount of work provided by the ventricle to induce flow in the aorta. The time dependent $P_{\text {res }}(t)$ can be expressed as a function of volumetric compliance $C_{v}\left(=d V / d P_{r e s}\right)$, and changes in aortic compartment volume with respect to time $(d V / d t)$, yielding:

$$
\frac{d P_{\text {res }}(t)}{d t}=\frac{d P_{\text {res }}(t)}{d V} \frac{d V}{d t}=\frac{1}{C_{V}}\left(Q_{\text {in }}(t)-Q_{\text {out }}(t)\right)
$$

where $Q_{\text {in }}(t)$ and $Q_{\text {out }}(t)$ are flow entering aortic compartment from the left ventricle and flow leaving the aortic compartment, respectively. The theory also describes the proportionality between each pressure component and flow dynamics in the aorta:

$$
\begin{aligned}
& P_{\text {res }}(t)-P_{\text {cvp }}=R Q_{\text {out }}(t) \\
& P_{\text {ex }}(t)=Z_{a o} Q_{\text {in }}(t)
\end{aligned}
$$


where $R, Z_{a o}$ and $P_{c v p}$ are peripheral resistance, aortic characteristic impedance and central venous pressure, respectively. In this analysis, $P_{c v p}$ was assumed $8 \mathrm{mmHg}$ for all the pigs as a typical value that could also be measured. Substituting Eqs. (5) and (6) into Eq. (4), the differential equation for reservoir pressure can be written.

$$
\frac{d P_{r e s}(t)}{d t}=\frac{P_{e x}(t)}{Z_{a 0} C_{V}}-\frac{P_{r e s}(t)-P_{c v p}}{R C_{V}}
$$

Assuming that aortic volumetric compliance $C_{v}$ can be written in the form $C_{v}=C_{A} L_{a 0}$, where $C_{A}$ and $L_{a o}$ are compliance per unit length of aorta and aortic length, respectively, and PWV can be described by aortic characteristic impedance and compliance per unit length [26], Eq. (7) can be rewritten;

$$
\begin{aligned}
& P W V=1 / Z_{a o} C_{A} \\
& \frac{d P_{\text {res }}(t)}{d t}=\frac{P W V}{L_{a o}} P_{e x}(t)-\frac{P_{r e s}(t)-P_{c v p}}{R C_{V}}
\end{aligned}
$$

By substituting Eq. (3) into Eq. (9) and applying the initial condition that $P_{\text {res }}(0)=$ $P_{\text {measured }}(0)$ at the beginning of a heartbeat, Eq. (9) can be solved for $P_{\text {res }}(t)$ over one cardiac cycle:

$$
P_{\text {res }}(t)=e^{-(\alpha+\beta) t}\left(\int_{0}^{t} e^{(\alpha+\beta) t^{\prime}}\left(\alpha P_{\text {measured }}\left(t^{\prime}\right)+\beta P_{\text {cvp }}\right) d t^{\prime}+P_{\text {measured }}(0)\right)
$$

where $\alpha$ and $\beta$ are $P W V / L_{a o}$ and $1 / R C_{v}$ respectively.

\section{Parameter Identification}

Parameter values $L_{a o}$ and $\beta$ were identified from the measured pressure waveform. In diastole, the measured pressure waveform decay can be assumed to result only from the release of energy stored by aortic compliance during systole. Thus, $P_{\text {measured }}$ represents $P_{\text {res }}$ in diastole, yielding:

$$
P_{\text {measured }}(t d<t<t f)=P_{\text {res }}(t d<t<t f)
$$

where $t d$ and $t f$ are the time at the start of diastole and end of diastole, respectively.

By performing grid search for $L_{a o}$ and $\beta$, the discrepancy between Eq. (10) and measured diastolic pressure decay was minimized. An example of the error surface produced from a grid search for a single beat is shown in Fig. 4. It can be seen in Fig. 4 that the surface is convex so an optimal value of $\beta$ could be identified for each value of $L_{a 0}$.

To identify the most suitable set of parameters, $L_{a o}$ and $\beta$, from the sets of optimal parameters identified by grid search, further constraints were added to the ESP to improve practical identifiability [27]. At end systole, ventricular hydraulic force equals aortic reservoir force pushing against the ventricle and thus, initiates closure of aortic valve. The flow entering into aorta from the ventricle would be zero at this point and, consequently, the excess pressure would be zero. Implementing this condition, the discrepancy between calculated end systolic pressure using the identified sets of $L_{a 0}$ and $\beta$ and measured end systolic pressure were minimized to select an optimal set of 


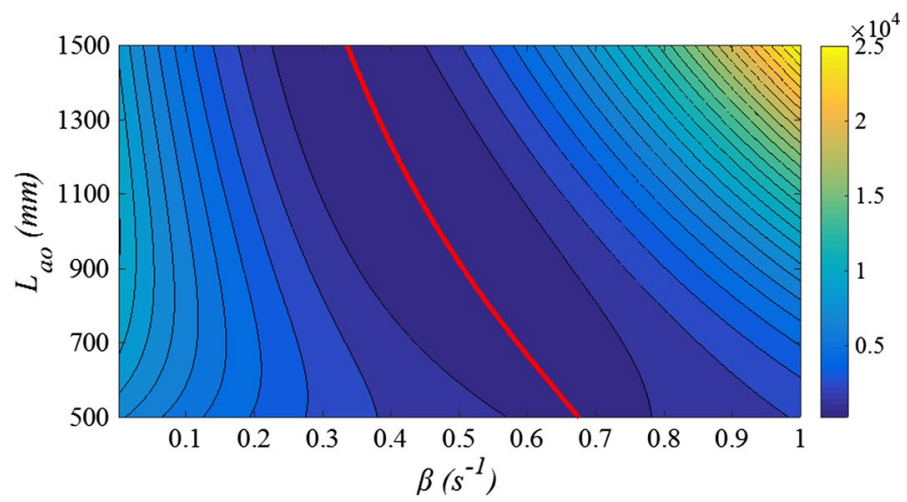

Fig. 4 Error surface showing the discrepancy between $P_{\text {measured }}\left(t d<t<t f\right.$ ) and calculated $P_{\text {res }}$ using different value of $L_{a o}$ and $\beta$ in Eq. 7. The red line represent optimal parameter $\beta$ for a given $L_{a o}$

parameters, $L_{a o}$ and $\beta$, for a given pressure waveform. An example of error curve for this optimization process is shown in Fig. 5.

The parameter identification process described above was used for the first 10 beats of the experiment for each pig. Once 10 values of $L_{a o}$ were identified for each pig, the values were averaged to give a representative $L_{a o}$ for each pig over the rest of the study. To solve Eq. (10), $L_{a o}$ was held constant because this anatomical length is not expected to change.

Using this fixed subject/pig-specific representative value of $L_{a 0}, \beta$ was optimized using the condition defined in Eq. (11) for each heart beat and pressure waveform. The calculated $P_{\text {res }}$ for each pressure waveform were then used to determine $P_{e x}$ and, subsequently, used to calculate aortic flow and SV.

\section{Stroke volume estimation}

The water hammer equation can be used to describe the relationship between PWV, changes in excess pressure $\left(d P_{e x}\right)$, and changes in velocity of blood through the aorta $\left(d U_{a o}\right)[28]$.

$$
d P_{e x}=\rho P W V d U_{a o}
$$

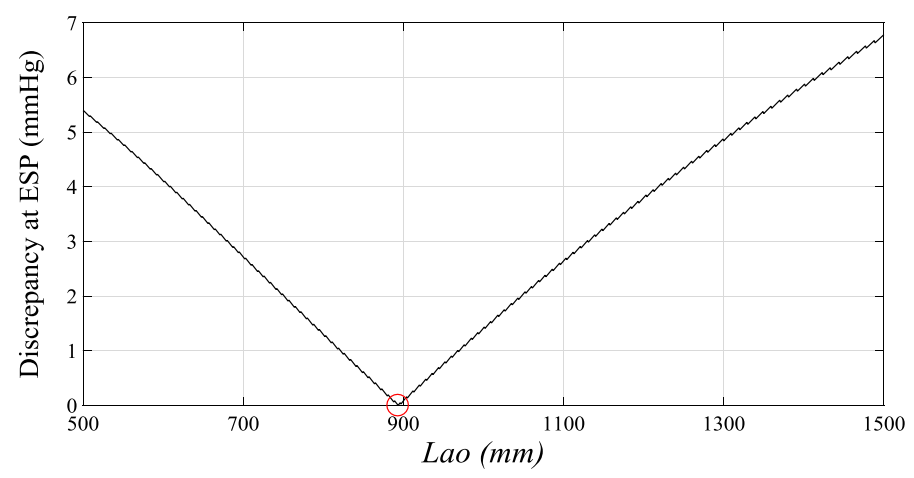

Fig. 5 The error curve showing the discrepancy between $P_{\text {measured }}$ at ESP and calculated $P_{\text {res }}$ at ESP using optimal sets of $L_{a o}$ and $\beta$ identified by grid search. The red circle shows the identified optimal parameter $L_{a 0}$ for a given pressure waveform 
where $\rho$ is the density of blood and assumed constant at $1050 \mathrm{~kg} / \mathrm{m}^{3}$ [29]. Taking $Q_{i n}=U_{a o} A_{a o}$ and substituting Eq. (6) into (12), the aortic characteristic impedance, $Z_{a o}$ can be expressed in terms of PWV, $\rho$, and $A_{a o}$.

$$
Z_{a o}=\rho P W V / A_{a o}
$$

For this analysis, the first 10 beats of measured left ventricular SV values from the admittance catheter were used to calibrate $A_{a o}$ for each pig. Identified $P_{e x}$ and measured $\mathrm{SV}=\int_{0}^{t_{d}} Q_{i n}$ were substituted into Eq. (6) to calculate $Z_{a o}$. Using these $Z_{a o}$ values, $A_{a o}$ were determined from Eq. (13). The average value of $A_{a o}$ for the first 10 beats was used to estimate SV.

\section{Relationship between model derived $A_{a o}$ and systolic period}

To capture the relative change in values of $A_{a o}$ over the course of an experiment, the relationship identified between $A_{a o}$ calculated using the measured SV and systolic period identified from pressure waveform were used to improve the estimate of $A_{a 0}$. Figure 6 shows the regression line minimizing the geometric mean deviation between relative changes in these two parameters for all pigs in relation to calibrated point. Regression using geometric mean deviation produces larger error in the estimated $A_{a o}$ from the relationship, however, this approach is more appropriate for this analysis as measurement error is expected in both parameters.

The relationship showed nearly 1:1 ratio in relative change between $A_{a o}$ and systolic period. This relationship was applied to calibrated $A_{a o}$ to identify a more accurate values of $Z_{a 0}$.

$$
\begin{aligned}
& \frac{A_{a o}}{A_{a o, 0}}=\frac{\text { Systolic Period }_{0}}{\text { Systolic Period }} \\
& Z_{a o}=\frac{\rho P W V}{A_{a o, 0}} \frac{\text { Systolic Period }}{\text { Systolic Period } 0}
\end{aligned}
$$

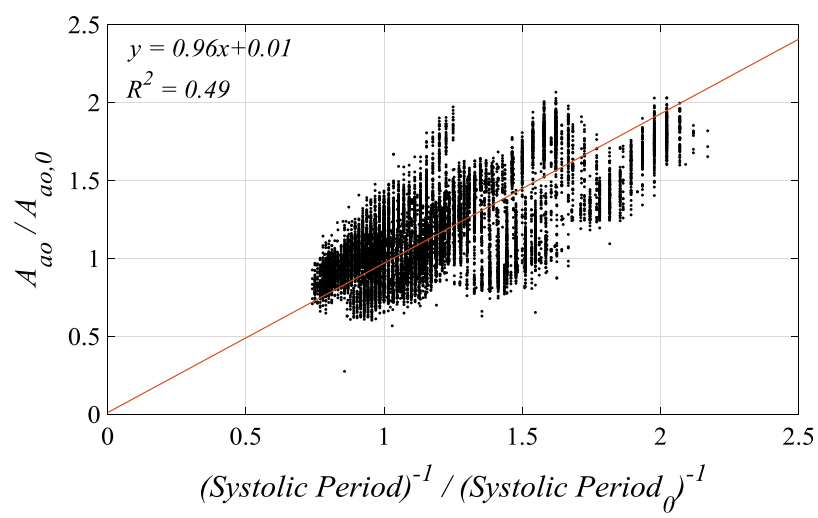

Fig. 6 Correlation plot showing relationship between relative change in aortic area $A_{a o}$ and systolic period

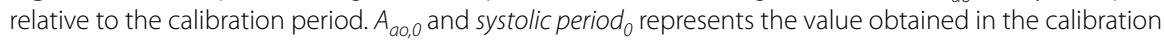
period 
where the subscript zero refers to calibration period, where $A_{a o}$ was obtained using 10 heart beats.

\section{Stroke volume}

The calculated aortic characteristic impedance from Eq. (15) and $P_{e x}$ using Eqs. (3) and (10) were then used to estimate SV.

$$
S V_{\text {estimate }}=\frac{1}{Z_{a o}} \int_{0}^{t f} P_{e x}(t) d t
$$

The SV values estimated using Eq. (16) are compared against measured SV from the admittance catheter using Bland-Altman plots. The schematic of processes involved in the pressure contour method are outlined in Appendix.

\section{Results}

The identified values of $L_{a 0}, A_{a 0,0}$, relevant physiological parameters, total number of heart beats analysed, and weight for each pig are shown in Table 2. Bland-Altman plots comparing SV estimated using Eq. (16) and SV measured from the admittance catheter are presented in Fig. 7. The summary of bias, 95\% interval, and precision calculated as half of $95 \%$ range divided by mean SV for each pig are shown in Table 3. In addition, time series showing measured and estimated SV in the last RM period, the most distant point from calibration and thus a potential worst case, for each pig are shown in Fig. 8.

Table 2 Summary of identified parameters $L_{a o}, A_{a o, 0}$ for all pigs and ranges of physiological parameters, mean aortic pressure (MAP), PWV, systolic period, and measured SV for volume expansion and dobutamine period

\begin{tabular}{|c|c|c|c|c|}
\hline & Pig 1 & Pig 2 & Pig 3 & Pig 4 \\
\hline \multicolumn{5}{|l|}{ Weight (kg) } \\
\hline & 24.5 & 20 & 23.5 & 23.3 \\
\hline \multicolumn{5}{|c|}{ Identified aortic dimension } \\
\hline $\mathrm{L}_{-}{ }_{\mathrm{ao}}(\mathrm{m})$ & 0.88 & 0.41 & 0.89 & 0.91 \\
\hline$A_{a o, 0}\left(m^{2}\right)$ & 201 & 269 & 473 & 163 \\
\hline \multicolumn{5}{|l|}{ Volume expansion } \\
\hline $\mathrm{MAP}(\mathrm{mmHg})$ & 152 [136 to 182$]$ & 114 [93 to 136$]$ & 111 [93 to 132] & 55 [42 to 77$]$ \\
\hline $\mathrm{PWV}(\mathrm{m} / \mathrm{s})$ & $5.5[5.3$ to 6.0$]$ & $6.7[6.4$ to 6.9$]$ & 8.5 [8.2 to 8.9$]$ & $3.9[3.7$ to 4.1$]$ \\
\hline Heart rate (beats/min) & $64[62$ to 67$]$ & 86 [78 to 94] & 81 [73 to 88$]$ & $71[69$ to 74$]$ \\
\hline Systolic period (s) & 0.38 [0.33 to 0.46$]$ & $0.32[0.25$ to 0.39$]$ & $0.31[0.26$ to 0.38$]$ & $0.33[0.31$ to 0.36$]$ \\
\hline $\mathrm{SV}_{\text {measured }}(\mathrm{ml})$ & 34 [28 to 40] & 19 [16 to 23$]$ & 30 [26 to 39] & 29 [25 to 34$]$ \\
\hline \multicolumn{5}{|c|}{ Dobutamine/+ volume expansion } \\
\hline Pressure $(\mathrm{mmHg})$ & $150[123$ to 184$]$ & $90[54$ to 124$]$ & 100 [79 to 123$]$ & 61 [43 to 95] \\
\hline $\operatorname{PWV}(\mathrm{m} / \mathrm{s})$ & $6.0[5.6$ to 6.7$]$ & $6.3[5.4$ to 6.8$]$ & $8.2[7.6$ to 8.8$]$ & $4.6[4.3$ to 5.2$]$ \\
\hline Heart rate (beats/min) & 104 [90 to 108] & $141[136$ to 142$]$ & 109 [98 to 120$]$ & $107[93$ to 115$]$ \\
\hline Systolic period (s) & $0.23[0.22$ to 0.31$]$ & 0.19 [0.18 to 0.22$]$ & $0.23[0.19$ to 0.3$]$ & $0.23[0.21$ to 0.31$]$ \\
\hline$S V_{\text {meansreud }}(\mathrm{ml})$ & 28 [23 to 36$]$ & $24[17$ to 27$]$ & 25 [21 to 28$]$ & $27[22$ to 31$]$ \\
\hline \multicolumn{5}{|c|}{ Total no of heart beats analysed } \\
\hline & 2956 & 4945 & 3057 & 2451 \\
\hline
\end{tabular}

Data are presented as the mean [5th-95th percentiles] 
$\mathbf{a}$

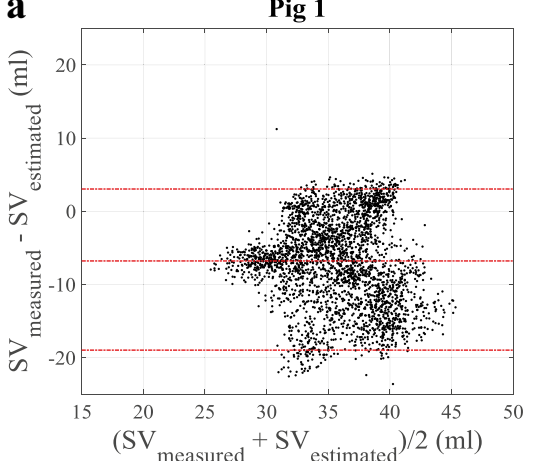

b

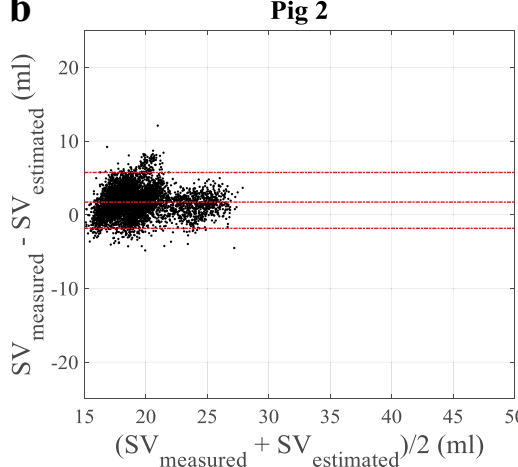

c

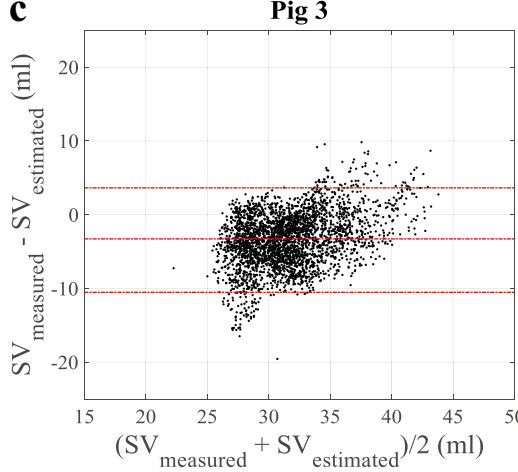

d

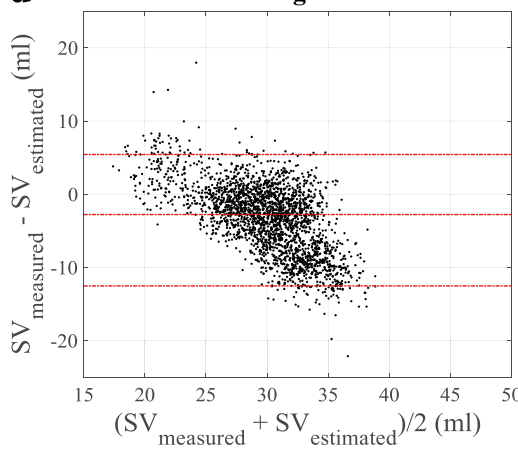

Error Distribution

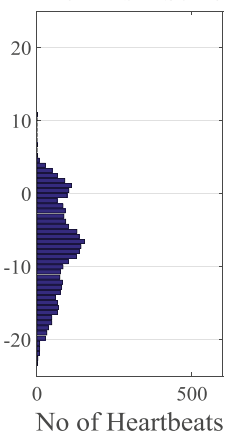

Error Distribution

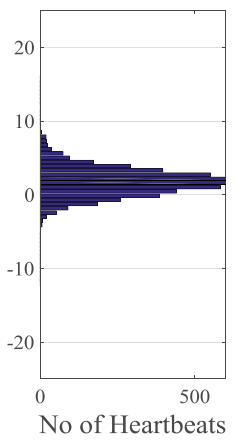

Error Distribution

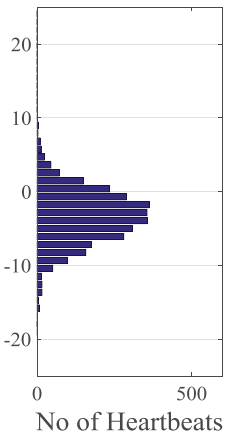

Error Distribution

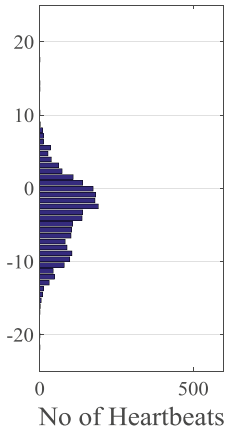

Fig. 7 Bland-Altman plots $\mathbf{a}-\mathbf{d}$ showing agreements between measured and estimated SV for all pigs. Red dashed line showing the bias and 95\% interval. Right panel showing the error distribution between measured and estimated SV values 
Table 3 Summary of Bland-Altman analysis for each pigs

\begin{tabular}{lll}
\hline Pig no. & Bland-Altman results $(\mathbf{m l})$ & Precision (\%) \\
\hline Pig 1 & $-6.8[-18.9$ to 3.0$]$ & 31 \\
Pig 2 & $1.7[-1.8$ to 5.7$]$ & 20 \\
Pig 3 & $-3.3[-10.5$ to 3.6$]$ & 22 \\
Pig 4 & $-2.8[-12.5$ to 5.4$]$ & 30 \\
\hline
\end{tabular}

Data are presented as the bias [2.5th-97.5th percentiles], where bias is the mean difference between measured and estimated SV. Precision is calculated as half of $95 \%$ range divided by mean SV for each pig

\section{Discussion}

To investigate the accuracy of the method, wide ranges of physiological conditions were analysed involving significant changes in heart rate, preload, afterload, and contractility of the heart. Fluid state and airway pressure were altered to induce preload changes producing more than 30 percent difference in SV. Dobutamine was infused to induce afterload and contractility changes involving decrease in ventricular-arterial coupling [30]. In addition, dobutamine increased heart rate by $50 \%$ in all pigs as can be seen in Table 2 .

The Bland-Altman results in Fig. 7 and Table 3 demonstrate the ability of the method in capturing the absolute value of SV. Despite all the significant hemodynamic changes, $95 \%$ range was within $\pm 10 \mathrm{ml}$ (approximately $\pm 30 \%$ ) for all pigs and many errors were much smaller. The method showed it is capable of estimating clinically acceptable absolute SV values according to Critchely and Critchely [31], where they states acceptable accuracy of the stroke volume estimation method against the reference method to be within approximately $\pm 30 \%$.

The ability of the method to capture SV trend/dynamics is shown in Fig. 8. The figure shows the last RM period of the experiment where one would expect to find the largest difference in the hemodynamic conditions. It can be seen that PEEP induced stroke volume trends were correctly captured in most cases. The method was able to capture both 'affected' (Pig 1, 2, and 4) and 'unaffected' (Pig 3) cases, which has clinical importance in preload assessment [32]. In addition, Stroke Volume Variability (SVV) induced from individual breath (cyclic effect) was correctly captured.

It can be noted that the method over-estimated the trend for Pig 4 and made incorrect estimation for Pig 1 during PEEP reduction. Pig 4 had an extremely low blood pressure, of approximately $45 \mathrm{mmHg}$ at the beginning of the experiment where the method was calibrated and had the biggest change in the mean pressure during the experiment, as can be seen in Table 2 (almost a twofold increase). In such a case, the method was not able to capture the dramatic change in aortic dimension and therefore, deviated from true value of SV. The error seen in Pig 1 was not able to be resolved as pressure and PWV values returned to normal after RM, similar to starting point of RM. However, the value of SV remained at a reduced value. In both cases, further investigations are necessary and there are potential to further improve the method.

\section{Pressure contour analysis}

There are several methods currently available for estimating continuous SV by pressure contour analysis [33]. In general, the Windkessels models are used and parameters involved in the model are identified from the single pressure waveform and/or patients' 

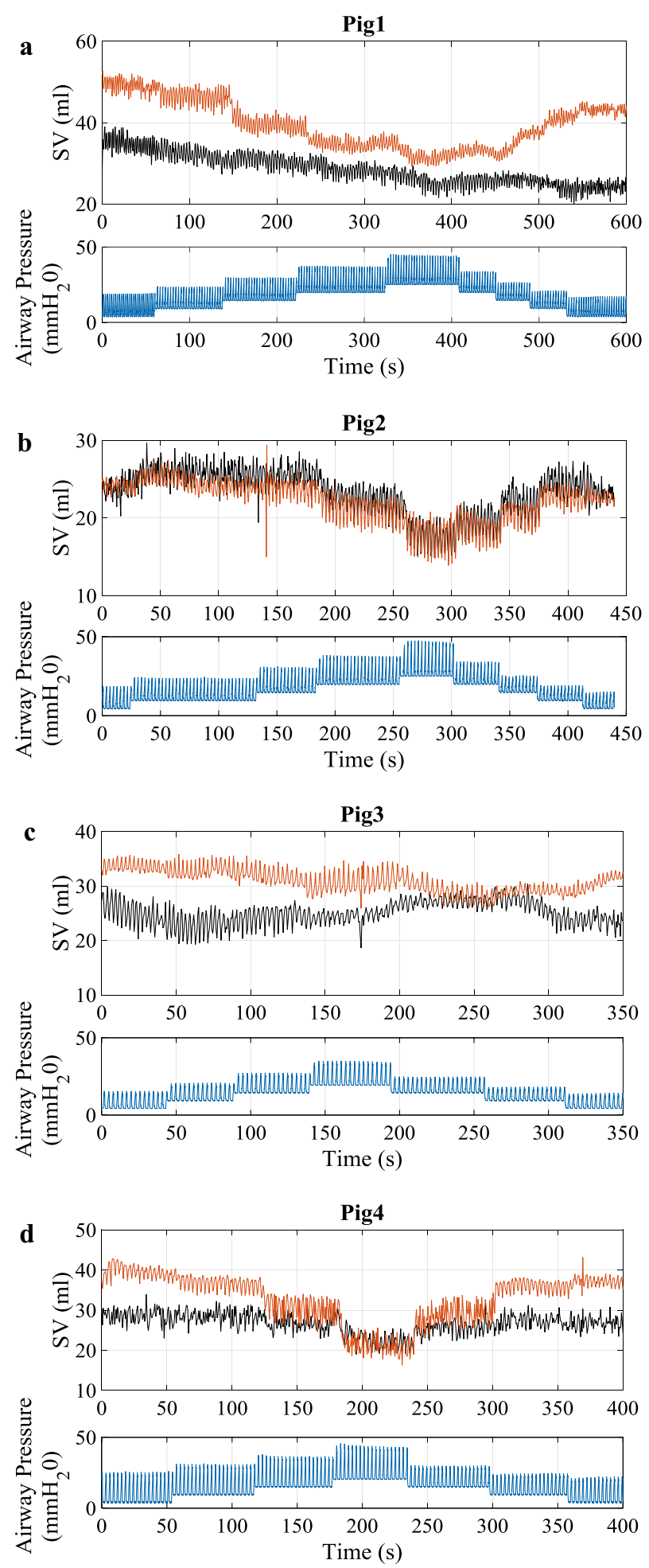

Fig. 8 Time series plot $\mathbf{a}-\mathbf{d}$ showing measured and estimated SV in the last RM period of the experiment for all pigs. Top panel measured SV from admittance catheter (black line) and estimated SV using Eq. (13) (red line). Bottom panel simultaneously measured airway pressure showing PEEP changes during recruitment manoeuvers RM (blue line) 
demographic/physical characteristics [34]. The significant improvement of the presented method over conventional methods is that PWV measurements are integrated into pressure contour analysis to accurately capture the dynamics of time-varying model parameters, which are needed to obtain SV correctly.

Pulse wave velocity measurements are related to arterial distensibility [35] and its relative changes within a subject provide additional information on the cause of changes in the characteristics of the pressure waveform. A combination of changes in the value of PWV and changes in the shape of diastolic pressure decay for a given aortic dimension gives a better approximation of the time-varying arterial reservoir function. The reservoir pressure waveform estimates the minimum pressure that ventricle must provide to induce flow into the artery [36] and is closely related to arterial impedance [37]. By assigning the correct components of the pressure waveform to each of the Windkessel parameters, and with added data inputs, the method is capable of capturing accurate physiological conditions and its changes.

\section{Estimation of aortic dimension}

In this work, the aortic dimension was estimated by assuming the aorta behaved as a simple cylindrical tube having uniform properties and pressure along it. In reality, the properties are non-uniform and each segment of aorta has different pressure contours. However, to obtain such a large amount of information in a clinical settings is impractical, if not impossible. These assumptions thus produce error in the estimation of SV, but are necessary in developing a method based on clinically accessible and reasonable measurements.

The identified values of $L_{a o}$ and calibrated values of $A_{a o}$ for each pig are shown in Table 2. These values represent an estimate of aortic dimensions for a given value of pressure contour and PWV. It is largely affected by the absolute value of PWV and SV values used to calibrate the method. Since even the reference values are expected to have error of approximately $\pm 20 \%$ [38], the identified values of aortic dimension may not be entirely realistic. However, given the large errors of reference values, the resulting values are of use, as are the resulting SV values.

The important part of this calibration is to give an estimate of the dimension under given conditions and to use the relative changes in the measurements to track the trends of hemodynamic parameters. In a clinical environment, the absolute value of SV is not of great importance. However, the relative changes in SV for different patient conditions and in response to therapy is of major clinical significance [39]. From this point of view, the method has shown the ability to sufficiently track the trends of SV (Fig. 8) under the assumptions made.

\section{Relationship between model derived $A_{a o}$ and systolic period}

The identified relationship shown in Fig. 6 uses $A_{a o}$ derived from Eq. (13) using the measured value of SV. In the experiment, true aortic diameter was not measured and consequently, the exact physiological reason behind the relationship between aortic area and systolic period was not able to be determined. This limitation is due to the possibility that this relationship may represent correlation between systolic periods with other physiological variables. 
Previous studies have identified the relationship between heart rate/ejection time and aortic PWV [40, 41]. The relationship can be explained by stiffening of the aortic wall due to viscoelastic properties. Equation (13), which is derived from the water hammer equation, fails to describe the energy loss due to hysteresis of the aorta and could thus have produced incorrect values of $A_{a 0}$.

To identify the exact source of the relationship presented in Fig. 6, accurate measurements of time-varying aortic area are required. Such measurements will produce a stress-strain relationship explaining the time-dependent mechanisms associated with arterial distensibility/stiffness. However, whether the relationship is due to the viscoelastic properties or not, the identified population relationship is still useful in providing an accurate estimation of SV and thus, it is used for the method presented until better data is available in subsequent experiments.

\section{Extending the method to alternative PWV estimation}

The experiments used here involved highly invasive method to obtain PWV, requiring two pressure measurements along aorta, which is uncommon in the critical care environment. However, the results from this analysis demonstrates the possibilities for more accurate estimation of SV if PWV can be estimated by this or different approach. The aortic arch pressure measurement was used only for the purpose of identifying the transit time and the information from its contour was not used. Therefore, this measurement can potentially be replaced with less invasive measurements such as ECG to detect another pulse location [42]. Previous studies show evidence for strong relationship between invasive and non-invasive PWV measurements [43, 44], and thus the method is expected to have negligible influence from use of alternative PWV measurements.

\section{Limitations}

The clinical applicability of the method presented is limited to the patient having at least one pressure measurement from a catheter in a central artery. Pressure sensing lumens in radial artery are commonly used in ICU patients. However, due to the current perceived risk-to-benefit ratio for having central pressure waveform, it is rarely used in clinical practice [45]. The ability and improved accuracy of estimating SV provided by the method may reverse this trend turning potential risk into benefit [46, 47]. Furthermore, identified time-varying Windkessel parameters (aortic characteristic impedance/compliance and systemic resistance) provides further insight into optimization of clinical treatment [48].

Another limitation of this study is that experiments were performed on healthy pigs and more complicated situations involving valve regurgitation, aneurysm, and/or any arterial defect were not analysed. Irregular pressure contours produced by these conditions could affect the parameter identification process and precision. To investigate the clinical applicability of the method in such cases, further validation and analysis must be done covering wider range of cardiovascular systems and circulatory dysfunctions.

\section{Conclusion}

The method presented in this paper accurately estimates and tracks SV trends even when hemodynamic properties are significantly altered. PWV measurements, which are usually available in the ICU or can easily be obtained non-invasively, were integrated 
into pressure contour analysis to overcome weaknesses associated with conventional methods for estimating SV. The additional information gained by PWV allowed precise estimation of Windkessel parameters and thus, accurate estimation of SV. The BlandAltman plots showed average $95 \%$ limits of agreement of $\pm 26 \%$ between estimated SV and the reference SV across all pigs, demonstrating the clinical applicability of the method. In addition, the method require only one calibration per subject making the method more practical. The method presented can track accurate and clinically important changes in patients' haemodynamic state providing essential information for correct diagnosis and optimal care.

\section{Authors' contributions}

Developed the model, analysed the data, and wrote the manuscript: SK. Designed and performed the experiments including data acquisition: SK, AP, TD. All authors were involved in the interpretation of the data and made contributions through the entire process. All authors read and approved the final manuscript.

\section{Author details}

${ }^{1}$ Department of Mechanical Engineering, University of Canterbury, Christchurch, New Zealand. ${ }^{2}$ GIGA Cardiovascular Science, University of Liege, Liege, Belgium. ${ }^{3}$ Intensive Care Unit, Christchurch Hospital, Christchurch, New Zealand.

\section{Acknowledgements}

Not applicable.

\section{Competing interests}

The authors declare that they have no competing interests.

\section{Ethics approval}

All experimental procedures, protocols and the use of data in this study were reviewed and approved by the Ethics Committee of the University of Liege Medical Faculty.

\section{Data availability}

The datasets generated during and/or analysed during the current study are available from the corresponding author on reasonable request.

\section{Funding}

NZ Tertiary Education Commission (TEC) fund MedTech CoRE (Centre of Research Expertise).

EU FP7 and RSNZ Marie Curie IRSES.

Health Research Council (HRC) of New Zealand. 


\section{Appendix}

See Fig. 9.

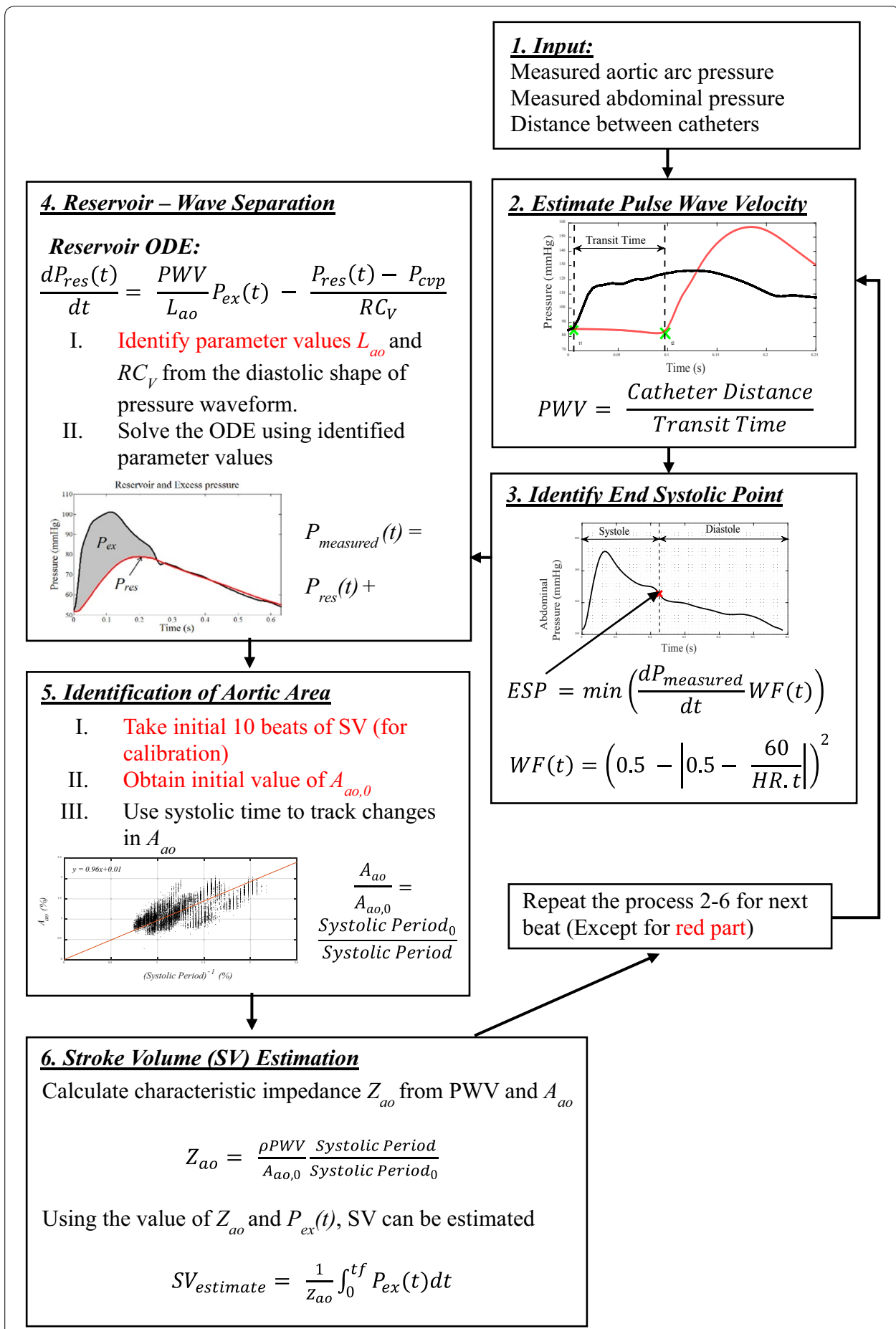

Fig. 9 Schematic of SV estimation processes showing key steps involved in the pressure contour method 


\section{Publisher's Note}

Springer Nature remains neutral with regard to jurisdictional claims in published maps and institutional affiliations.

Received: 17 November 2016 Accepted: 19 April 2017

Published online: 24 April 2017

\section{References}

1. Rivers $\mathrm{E}$, et al. Early goal-directed therapy in the treatment of severe sepsis and septic shock. N Engl J Med. 2001;345:1368-77.

2. Beal AL, Cerra FB. Multiple organ failure syndrome in the 1990s: systemic inflammatory response and organ dysfunction. JAMA. 1994;271(3):226-33.

3. Marik PE, Monnet $X$, Teboul J-L. Hemodynamic parameters to guide fluid therapy. Transfus Altern Transfus Med. 2010;11(3):102-12.

4. Ellender TJ, Skinner JC. The use of vasopressors and inotropes in the emergency medical treatment of shock. Emerg Med Clin North Am. 2008:26(3):759-86.

5. Tibby SM, Murdoch IA. Monitoring cardiac function in intensive care. Arch Dis Child. 2003;88:46-52.

6. Cecconi M, et al. Consensus on circulatory shock and hemodynamic monitoring. Task force of the European Society of Intensive Care Medicine. Intensive Care Med. 2014;40(12):1795-815.

7. Porhomayon J, et al. Cardiac output monitoring devices: an analytic review. Intern Emerg Med. 2012:7(2):163-71.

8. Alhashemi JA, Cecconi M, Hofer CK. Cardiac output monitoring: an integrative perspective. Crit Care. 2011;15(2):214

9. Lefrant J, et al. Training is required to improve the reliability of esophageal Doppler to measure cardiac output in critically ill patients. Intensive Care Med. 1998;24(4):347-52.

10. Critchley $L$, et al. The effect of lung injury and excessive lung fluid, on impedance cardiac output measurements, in the critically ill. Intensive Care Med. 2000;26(6):679-85.

11. Bein B, et al. The reliability of pulse contour-derived cardiac output during hemorrhage and after vasopressor administration. J Am Soc Anesthesiol. 2007;107:107-13.

12. Bendjelid K. When to recalibrate the $\mathrm{PiCCO}^{\mathrm{TM}}$ ? From a physiological point of view, the answer is simple. Acta Anaesthesiol Scand. 2009:53(5):689-90.

13. Groeneveld AJ, Polderman K. Fluid resuscitation: the good, the bad and the ugly. Crit Care Shock. 2005;8(1):52-4.

14. Overgaard CB, Dzavik V. Inotropes and vasopressors - review of physiology and clinical use in cardiovascular disease. Circulation. 2008;118(10):1047-56.

15. Shi Y, Lawford P, Hose R. Review of zero-D and 1-D models of blood flow in the cardiovascular system. BioMedical Eng Online. 2011;10(1):33.

16. Tyberg JV, et al. The case for the reservoir-wave approach. Int J Cardiol. 2014;172(2):299-306.

17. Wesseling $\mathrm{K}$, et al. Computation of aortic flow from pressure in humans using a nonlinear, three-element model. J Appl Physiol. 1993;74:2566-73.

18. Langewouters G, Wesseling K, Goedhard W. The static elastic properties of 45 human thoracic and 20 abdominal aortas in vitro and the parameters of a new model. J Biomech. 1984;17(6):425-35.

19. Kamoi $\mathrm{S}$, et al. Continuous stroke volume estimation from aortic pressure using zero dimensional cardiovascular model: proof of concept study from porcine experiments. PLOS ONE. 2014;9(7):e102476.

20. Slifka MK, Whitton JL. Clinical implications of dysregulated cytokine production. J Mol Med. 2000;78(2):74-80.

21. Camporota L, Beale R. Pitfalls in haemodynamic monitoring based on the arterial pressure waveform. Crit Care. 2010;14(2):124.

22. Luecke T, Pelosi P. Clinical review: positive end-expiratory pressure and cardiac output. Crit Care. 2005;9(6):607-21.

23. Koganov Y, et al. Positive end-expiratory pressure increases pulmonary venous vascular resistance in patients after coronary artery surgery. Crit Care Med. 1997;25(5):767-72.

24. Abel FL. Maximal negative $\mathrm{dP} / \mathrm{dt}$ as an indicator of end of systole. Am J Physiol Heart Circ Physiol. 1981;240(4):H676-9.

25. Wang J-J, et al. Time-domain representation of ventricular-arterial coupling as a windkessel and wave system. Am J Physiol Heart Circ Physiol. 2003;284(4):H1358-68.

26. Hughes $A D$, Parker $\mathrm{KH}$. Forward and backward waves in the arterial system: impedance or wave intensity analysis? Med Biol Eng Comput. 2009:47(2):207-10.

27. Pironet A, et al. Structural identifiability analysis of a cardiovascular system model. Med Eng Phys. 2016;38(5):433-41.

28. Hanya S. Validity of the water hammer formula for determining regional aortic pulse wave velocity: comparison of one-point and two-point (Foot-to-Foot) measurements using a multisensor catheter in human. Ann Vasc Dis. 2013;6(2):150.

29. Trudnowski RJ, Rico RC. Specific gravity of blood and plasma at 4 and $37^{\circ} \mathrm{C}$. Clin Chem. 1974;20(5):615-6.

30. Ruffolo RR Jr. The pharmacology of dobutamine. Am J Med Sci. 1987;294(4):244-8

31. Critchley LA, Critchley JA. A meta-analysis of studies using bias and precision statistics to compare cardiac output measurement techniques. J Clin Monit Comput. 1999;15(2):85-91.

32. Monnet $X$, Teboul J-L. Assessment of volume responsiveness during mechanical ventilation: recent advances. Crit Care. 2013;17(2):217

33. Montenij LJ, de Waal EEC, Buhre WF. Arterial waveform analysis in anesthesia and critical care. Curr Opin Anesthesiol. 2011;24(6):651-6.

34. Thiele RH, Durieux ME. Arterial waveform analysis for the anesthesiologist: past, present, and future concepts. Anesth Analg. 2011;113(4):766-76.

35. Bramwell JC, Hill AV. The velocity of the pulse wave in man. Proc R Soc London Series B Contain Papers Biol Character. 1922;93(652):298-306 
36. Parker K, Alastruey J, Stan G-B. Arterial reservoir-excess pressure and ventricular work. Med Biol Eng Comput. 2012;50(4):419-24.

37. Milnor WR. Arterial impedance as ventricular afterload. Circ Res. 1975;36(5):565-70.

38. Peyton PJ, Chong SW. Minimally invasive measurement of cardiac output during surgery and critical careA metaanalysis of accuracy and precision. J Am Soc Anesthesiol. 2010;113(5):1220-35.

39. Marik PE. Noninvasive cardiac output monitors: a state-of the-art review. J Cardiothorac Vasc Anesth. 2013;27(1):121-34.

40. Lantelme $P$, et al. Heart rate an important confounder of pulse wave velocity assessment. Hypertension. 2002;39(6):1083-7.

41. Salvi P, et al. Left ventricular ejection time, not heart rate, is an independent correlate of aortic pulse wave velocity. J Appl Physiol. 2013;115(11):1610-7.

42. Loukogeorgakis $\mathrm{S}$, et al. Validation of a device to measure arterial pulse wave velocity by a photoplethysmographic method. Physiol Meas. 2002;23(3):581.

43. Horvath IG, et al. Invasive validation of a new oscillometric device (Arteriograph) for measuring augmentation index, central blood pressure and aortic pulse wave velocity. J Hypertens. 2010;28(10):2068-75.

44. Podolec P, et al. Aortic pulse wave velocity and carotid-femoral pulse wave velocity: similarities and discrepancies. Hypertens Res. 2007;30(12):1151-8.

45. Lorente L, et al. Arterial catheter-related infection of 2949 catheters. Crit Care. 2006;10(3):R3.

46. Benedetto $U$, et al. The impact of arterial cannulation strategy on operative outcomes in aortic surgery: evidence from a comprehensive meta-analysis of comparative studies on 4476 patients. J Thor Cardiovasc Surg. 2014;148(6):2936-43.

47. Cousins TR, O'Donnell JM. Arterial cannulation: a critical review. AANA J. 2004:72(4):267-71.

48. Westerhof N, Lankhaar JW, Westerhof BE. The arterial Windkessel. Med Biol Eng Compu. 2009;47(2):131-41.

Submit your next manuscript to BioMed Central and we will help you at every step:

- We accept pre-submission inquiries

- Our selector tool helps you to find the most relevant journal

- We provide round the clock customer support

- Convenient online submission

- Thorough peer review

- Inclusion in PubMed and all major indexing services

- Maximum visibility for your research

Submit your manuscript at www.biomedcentral.com/submit 